\title{
Competencias interculturales: Impacto del taller Comunicándonos en la sensibilidad intercultural de personal docente universitario
}

\author{
Intercultural Competences: Impacts of the Workshop "Communicating" on Intercultural \\ Sensitivity of University Teachers
}

\section{Competências Interculturais: Impacto da oficina Comunicando-nos na sensibilidade intercultural de professores universitários}

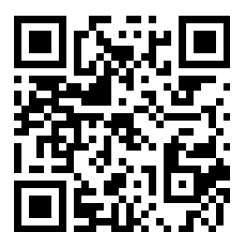

Carlos Enrique Ayala-Asencio

Universidad César Vallejo

Lima, Perú

carlosayala2105@gmail.com

https://orcid.org/0000-0003-4764-4359

Recibido • Received • Recebido: 06 / 01 / 2018

Corregido • Revised • Revisado: 16 / 06 / 2020

Aceptado • Accepted • Aprovado: 18 / 08 / 2020

\begin{abstract}
Resumen: La investigación tuvo por objetivo, conocer el impacto del taller "Comunicándonos" sobre las dimensiones de conciencia, habilidad y sensibilidad de las competencias interculturales del personal docente de un área académica profesional de una universidad privada de Lima - 2018, con la finalidad de desarrollar sus competencias interculturales contribuyendo en la formación docente, de tal forma que la enseñanza a desarrollar para el estudiantado logre los objetivos de calidad educativa. La metodología estuvo basada en el modelo positivista, de enfoque cuantitativo, de tipo aplicada, de diseño experimental-cuasi experimental. Utilizó la técnica de la encuesta y el instrumento del cuestionario. Se consideró como muestra a una no probabilística conformada por 40 docentes de universidad. Se procesó la información con apoyo de la estadística descriptiva e inferencial, con el fin de establecer cómo los datos cumplen, o no, con los objetivos de la investigación. La aplicación del referido taller causó impactos positivos en las competencias interculturales en las tres dimensiones materia de estudio, ya que los resultados estadísticos muestran que las diferencias de puntajes entre pretest y postest son significativos, comprobándose la teoría de Jandt (1995), en el sentido de que estas competencias se aprenden y desarrollan, entre ellas la sensibilidad intercultural del personal docente universitario.
\end{abstract}

Palabras claves: Competencias interculturales; conciencia; habilidad; sensibilidad. 
http://doi.org/10.15359/ree.24-3.18

http://www.una.ac.cr/educare

educare@una.ac.cr

\begin{abstract}
This research aimed to find out the impacts of the "Communicating" Workshop on the dimensions of consciousness, ability, and sensitivity of the intercultural competences of the teaching staff of a professional academic area of a private university in Lima - 2018, in order to develop their intercultural competences contributing to teacher training, in such a way that the teaching to be developed for the student body achieves the educational quality objectives. The methodology was based on the positivist model, with a quantitative approach, of the applied type, with an experimentalquasi-experimental design. He used the survey technique and the questionnaire instrument. A nonprobability sample made up of 40 university teachers was considered as a sample. Processing the information with the support of descriptive and inferential statistics in order to establish how the data does or does not meet the research objectives. The application of the aforementioned Workshop caused positive impacts on intercultural competences in the three dimensions studied, since the statistical results show that the differences in scores between pretest and posttest are significant, proving Jandt's Theory (1995) in the sense that These competencies are learned and developed, including the intercultural sensitivity of the university teacher.
\end{abstract}

Keywords: Intercultural competences; awareness; ability; sensitivity.

Resumo: O objetivo da pesquisa foi descobrir o impacto da Oficina "Comunicando-nos" nas dimensões de consciência, habilidade e sensibilidade das competências interculturais de docentes de uma área acadêmica profissional de uma universidade privada de Lima - 2018, a fim de desenvolver suas competências interculturais, contribuindo a formação de professores, de forma que o ensino a ser desenvolvido para estudantes atinja os objetivos de qualidade educacional. A metodologia foi baseada no modelo positivista, com abordagem quantitativa, do tipo aplicado, com delineamento experimental quase-experimental. Foi utilizada a técnica de perguntas e o instrumento do questionário. Como amostra não probabilística participaram 40 professores universitários. As informações foram processadas com o apoio de estatística descritiva e inferencial, a fim de estabelecer como os dados atendem ou não aos objetivos da pesquisa. A aplicação da oficina mencionada causou impactos positivos nas competências interculturais nas três dimensões estudadas, uma vez que os resultados estatísticos mostram que as diferenças nas pontuações entre pré-teste e pós-teste são significativas, comprovando a Teoria de Jandt (1995) no sentido de que essas competências são aprendidas e desenvolvidas, incluindo a sensibilidade intercultural de professores universitário.

Palavras-chave: Competências interculturais; consciencia; habilidade; sensibilidade.

\title{
Introducción
}

Un mundo globalizado en el cual se evidencia la diversidad cultural en un nivel intenso y dinámico exige que el personal docente universitario sea competente interculturalmente, para una adecuada interacción con el estudiantado. Fortalecer competencias interculturales en docentes en el nivel pregrado no solo implica aplicar conceptos básicos educativos, sino también la disposición docente expresada de manera pragmática, coherente y organizada en cada una de las interrelaciones, facilitando la mejora de la calidad educativa. 
http://doi.org/10.15359/ree.24-3.18

En un contexto globalizado, es preciso valorar el impacto de las diferencias entre las culturas en el contexto educativo que implica repensar planes, lineamientos y metas en el sistema educativo.

Las diferencias culturales no solo se evidencian en la relación docente-dicente, sino también en la relación entre el mismo estudiantado dentro y fuera del aula, tornándose cada vez más complejas.

El objetivo de la investigación fue determinar el efecto del taller "Comunicándonos" en las competencias comunicativas y en las competencias interculturales en el personal docente de un área académico profesional de una universidad privada de Lima, 2018.

El objetivo del presente artículo es la difusión del impacto de una investigación de diseño cuasiexperimental en las sensibilidades interculturales de competencias interculturales en el personal docente de un área académica profesional de una universidad privada de Lima, 2018.

\section{Estado de la cuestión}

Morales-Saavedra et al. (2018) en la investigación titulada: "Interculturalidad en educación superior: Experiencia en educación inicial en La Araucanía, Chile" por la Universidad Católica de Temuco de Chile, plantearon como objetivo lograr resultados respecto a la experimentación desempeñada en los procedimientos de formación inicial docente. Su metodología fue el enfoque mixto, con técnicas de encuesta e instrumentos de cuestionario y entrevista. Llegaron a concluir que existen aspectos relevantes para la formación de menores de seis años en el marco de una educación intercultural, en el cual son esenciales la comunicación, y el trabajo coordinado entre la familia y la comunidad. En el mismo sentido, Gastulo Jiménez et al. (2018), en la investigación titulada: "Modelo holístico configuracional para el desarrollo de la competencia intercultural en I docentes de educación secundaria en Motupe - Perú", plantearon como objetivo diseñar un modelo holístico configuracional sustentado en el enfoque intercultural y la teoría holística configuracional, para desarrollar la competencia intercultural. La metodología empleada fue de enfoque mixto y de diseño experimental. Aplicaron las técnicas de la encuesta y el análisis de datos y como instrumentos el cuestionario y la entrevista. Llegaron a la conclusión de que en el proceso formativo a docentes de educación secundaria de la institución educativa Cruz de Chalpón de Motupe existen deficiencias en el desarrollo de la competencia intercultural, lo que se manifiesta en el limitado desarrollo de los conocimientos, destrezas y actitudes interculturales, lo cual genera ausencia de conciencia cultural crítica. Por otro lado, resaltando la competencia intercultural, Martínez-Lirola (2018) en su investigación titulada: "La importancia de introducir la competencia intercultural en la educación superior: Propuesta de actividades prácticas" en la Universidad de Alicante, España, tuvo como objetivo incorporar el tema cultural por medio de la propuesta de actividades, tales como trabajar con textos multimodales de temática social en el proceso de enseñanza-aprendizaje, entre otras. Utilizó una metodología cooperativa, debido al 
http://doi.org/10.15359/ree.24-3.18

http://www.una.ac.cr/educare

educare@una.ac.cr

alto número de participantes en la investigación, asimismo se diseñó una encuesta que buscó la reflexión sobre la competencia intercultural, así como conocer su opinión acerca de ciertos tópicos vinculados con la interculturalidad. Llegó a concluir que es importante trabajar más actividades vinculadas con la convivencia entre personas de diferentes culturas, si es que el personal docente aspira a que la competencia intercultural ocupe un lugar prioritario en su quehacer docente. Por tanto, hay urgencia de cambios en la docencia como lo señala, Sanhueza Henríquez et al. (2016) en la investigación titulada: "Competencias comunicativas interculturales en la formación inicial docente: El caso de tres universidades regionales de Chile", en la Universidad Católica de Maule, Chile, propusieron como propósito el determinar el nivel de habilidades cognitivas, afectivas y comportamentales en estudiantes de las especialidades de educación parvularia y educación básica, con el fin de atender la diversidad sociocultural del futuro alumnado. La metodología utilizada fue el de enfoque cuantitativo y de diseño no experimental. Emplearon técnicas de la encuesta, la observación y el análisis de datos, cuestionarios y encuestas. Sus conclusiones indicaron sobre la necesidad de mejorar los currículos formativos del personal docente, mediante la inclusión de la diversidad cultural de modo integral.

Es decir, la importancia de las competencias interculturales en docentes es esencial en el proceso educativo, como lo sostiene Illescas García (2015) en su investigación titulada: "La competencia intercultural en la enseñanza de Español lengua extranjera: Trabajo de campo sobre manuales y estudiantes de español" para obtener el grado de doctor por la Universidad de Granada, España. El objetivo de su investigación fue relevar la importancia de la competencia intercultural en los estudios de segundas lenguas de estudiantes de lengua extranjera de la Universidad de Granada, y si es posible evaluar el nivel de competencia intercultural y establecer una correspondencia con el nivel de las demás competencias de la lengua que se aprende. La metodología empleada, en cuanto al enfoque, es cuantitativo, de diseño experimental. Aplicó las técnicas de encuesta y como instrumento el cuestionario. Llegó a concluir sobre la importancia del componente intercultural en la enseñanza de la segunda lengua con la finalidad que el alumnado puedan interactuar con los sujetos hablantes nativos en igualdad de condiciones; la premisa que lengua y cultura no se pueden disociar sigue siendo válida y quedó manifestado a lo largo del trabajo. En similar manera, lo afirma Guadamillas Gómez (2014) en su investigación titulada: El desarrollo de la competencia comunicativa e intercultural a través de recursos creativos: Análisis de la poesía, el teatroy el cómic como materiales didácticos en la enseñanza de inglés comolengua extranjera para obtener el grado de doctor por la Universidad de Castilla de la ciudad de la Mancha, España. El objetivo de su investigación fue proponer criterios válidos de análisis manuales de lengua inglesa, que puedan suponer una herramienta para conocer el potencial comunicativo e intercultural de actividades concretas en docentes de lengua inglesa. La metodología empleada, en cuanto al tipo de investigación, fue de enfoque mixto, de diseño experimental. Aplicó las técnicas de encuesta y entrevista y como instrumentos el cuestionario y la guía de entrevista. Llegó a la conclusión de afirmar la conexión de las teorías de enseñanza de la lengua inglesa con el potencial del uso de recursos creativos literarios en el incremento de una enseñanza comunicativa e intercultural. 
http://doi.org/10.15359/ree.24-3.18

\section{Marco teórico}

DeVallescar Palanca (2000) señala que la interculturalidad se caracteriza por ser un proceso de mestizaje por el cual cada persona y cada cultura cooperan al destino de la humanidad y el universo, el cual depende en buena parte de cada individuo. Desde un enfoque filosófico, Consorcio Intercultural (2007) indica que la interculturalidad supone diversidad y diferencia, diálogo y contraste, que suponen a su vez procesos de apertura, de indefinición e incluso de contradicción. A diferencia de la pluriculturalidad, la interculturalidad es todavía una apuesta por la universalidad. No renuncia a valores o normas universales. Al contrario, es característico del planteamiento intercultural buscar contenidos universalizables que permitan converger, por ejemplo, en el principio de que no es bueno matar o hacer daño a la otra persona. Con una perspectiva teológica, Estermann (2010) conceptualiza la pluriculturalidad con el hecho de la coexistencia de muchas o varias culturas en un mismo espacio geográfico o político, mientras que la interculturalidad es la relación entre dos o más culturas, a fin de enriquecerse mutuamente y contribuir a mayor plenitud humana. Por otro lado, desde un punto de vista pedagógico, Preiswerk (2011) conceptualiza la interculturalidad como un proyecto de convivencia, de interacción, intercambio y diálogo entre culturas dentro de un proceso mayor de transformación de las relaciones económicas, sociales y políticas. Por otro lado, distingue interculturalidad y pluriculturalidad, en el sentido de que la primera no se limita a reconocer las diferencias, a compararlas, a coleccionarlas, sino que las devela, les da valor, las pone en juego y las ubica en perspectiva. En este orden de ideas, considero pertinente manejar en este artículo los conceptos de pluriculturalidad e interculturalidad con rigurosa claridad, en primer lugar por ser conceptos polivalentes y convergentes; $y$, en segundo lugar, por significar procesos complejos que demandan responsabilidad a la humanidad.

Meyer (1991) define a la competencia intercultural, como parte de una amplia competencia de hablantes de una lengua extranjera, con la habilidad de una persona de actuar de forma adecuada y flexible al enfrentarse con acciones, actitudes y expectativas de personas de otras culturas. La adecuación y la flexibilidad implican poseer un conocimiento de las diferencias culturales entre la lengua extranjera y la propia; además, tener la habilidad de solucionar problemas interculturales como consecuencia de dichas diferencias. Por otro lado, Jandt (1995) define las competencias interculturales como la mixtura de diversas actitudes relacionadas con la diversidad cultural, competencias comunicativas, capacidad de manejar conflictos interculturales, auto conciencia cultural y como estas afectan en la cosmovisión de la realidad. Con una mirada pedagógica, Aneas Álvarez (2005) señala que los efectos de la competencia intercultural son diversos y abarcan dimensiones psicológicas, sociales o profesionales, tanto en el plano social como en el individual. Dichos efectos son la adaptación social, la integración cultural, el incremento de la idoneidad profesional y la salud psicológica, aspectos que influyen en el ejercicio de la ciudadanía. Con un enfoque de procesos, Salmona et al. (2015) definieron a las competencias interculturales como aquel que se aplica ampliamente como un proceso continuo de mejora de los conocimientos y habilidades que mejoran la interacción profesional 
http://doi.org/10.15359/ree.24-3.18

http://www.una.ac.cr/educare

educare@una.ac.cr

en situaciones interculturales. De mi parte, definir la competencia intercultural significa el esfuerzo personal por desarrollar capacidades logrando el equilibrio personal y luego cooperar en el proceso de interrelaciones culturales para que otras personas también se equilibren.

Byram (1997) define la competencia comunicativa intercultural como la habilidad de comprender y relacionarse con personas de otros países, y pretende ser una descripción completa y rica de lo que se requiere en las circunstancias más complejas y también más favorables de la comunicación intercultural. En similar sentido, Sanhueza Henríquez et al. (2012) indican que el modelo de competencia comunicativa intercultural diseñado por Chen y Starosta tiene como fundamento la pluriculturalidad. Señalan, además, que el modelo enfatiza acciones de reconocimiento, reverencia, flexibilidad e integración de las diferentes culturas, el cual configura una nueva ciudadanía. Asimismo, el modelo en referencia se explica en un procedimiento de transformación de interdependencia simétrica expresado en la conciencia intercultural, habilidad intercultural y sensibilidad intercultural. En mi criterio, la competencia comunicativa intercultural debe representar significancia en el proceso de formación de docentes hacia la calidad educativa intercultural, pues esta competencia potencializa nuevas formas de comunicación para enseñar.

Asimismo, Sanhueza Henríquez et al. (2012) exponen que la sensibilidad intercultural tiene una perspectiva afectiva, la cual se enfoca en emociones personales o ajustes de sentimientos causados por situaciones particulares entre personas y distintos ambientes. Por otro lado, la sensibilidad intercultural se puede explicar a través de indicadores tales como: (a) Autoconcepto, que indica la manera en que la persona se concibe a sí misma. (b) Apertura de mente, referido al reconocer, el apreciar y aceptar las iniciativas de otras culturas. (c) Actitudes de no juzgar, que señala las acciones de no adelantar ideas preconcebidas sino la disposición de una escucha activa en la comunicación intercultural. (d) Empatía, que indica la disposición de ubicarse en la persona de alguien culturalmente distinta, para así construir ideas y emociones interculturales. (e) La autorregulación, es la posibilidad de identificar acciones específicas para regularse y modificar comportamientos y, (f) Implicación en la interacción, característica que se vincula a la sensibilidad, atención y perspicacia.

\section{Metodología}

La metodología de investigación empleada fue: de paradigma positivista, de enfoque cuantitativo, de tipo aplicada y con enfoque cuantitativo. El diseño de investigación utilizado fue experimental-cuasi experimental. El estudio utilizó como variables: (a) Competencias comunicativas (dimensiones cognitiva, comunicativa y sociocultural). y (b) Competencias interculturales (dimensiones conciencia intercultural, habilidad intercultural y sensibilidad intercultural). Se trabajó con dos grupos de análisis, grupo experimental (GE), al cual se aplicó 
http://doi.org/10.15359/ree.24-3.18

el taller "Comunicándonos" y el grupo de control (GC), que no recibió ningún estímulo. Por otro lado, a los dos grupos les fueron administrados, en dos oportunidades (pretest y postest, sus aplicaciones transcurrieron en una semana), el instrumento "Cuestionario sobre el nivel de sensibilidad intercultural", se verificó así la equivalencia inicial de los grupos en referencia para la prueba pretest, mientras que en el postest si se observó efecto en la sensibilidad intercultural para el grupo experimental.

Para la obtención de la información se convocó a personal docente universitario, al cual se le aplicó el cuestionario, se les informó de su estructura y se le explicó, con un ejemplo, la manera de llenarlo de manera escrita y autoadministrada. La persona encuestada respondió sin intervención de ningún sujeto encuestador. El instrumento utilizado estuvo constituido por un conjunto de ítems de tipo opción múltiple que fueron medidos con una escala Likert de cinco puntos, de 1 a 5 ( 1 es nunca, 2 es casi nunca, 3 es algunas veces, 4 es casi siempre y 5 es siempre) (ver apéndice $\mathrm{A})$.

En el presente trabajo, la población estuvo enfocada en docentes de un área académica profesional de una universidad privada de Lima, cuya población es de cuarenta y cinco (45) docentes, de los cuales se seleccionó a cuarenta (40), para lo cual se consideró la disponibilidad. En el caso del grupo experimental se seleccionó el primer grupo de veinte (20) docentes que confirmaron su participación en el estudio y el siguiente grupo de veinte (20) conformó el grupo de control. El muestreo utilizado en la investigación fue no probabilístico, de tipo intencional (Arias-Gómez et al., 2016).

En esta investigación se aplicaron talleres, en consideración a que este es un espacio en el cual se facilita la enseñanza y aprendizaje, mediante la realización desde lo vivencial y no solo desde la transmisión. Se trata, entonces, de un aprender haciendo, donde los conocimientos se adquieren a través de una práctica concreta, realizando algo relacionado con la formación que se pretende proporcionar a sus participantes. Es una metodología participativa en la que se enseña y se aprende a través de una tarea conjunta (Careaga et al., 2006). Los talleres se realizaron en seis sesiones con una duración total de doce horas, en las cuales predominó la interacción práctica entre todos los grupos de docentes participantes con el monitoreo del equipo facilitador. El Apéndice B contiene el detalle de la temática efectuada en el taller "Comunicándonos", así como algunas actividades realizadas en este mismo.

La validez del instrumento de esta investigación se realizó mediante juicio experto, en el cual se seleccionaron cuatro personas expertas (equipos investigadores del área objeto del estudio y de metodología) quienes evaluaron, de manera independiente, la adecuación o ajuste de los ítems de los instrumentos.

Para el análisis de datos se usó el estadístico descriptivo de la comparación de frecuencia y porcentajes, y para el estadístico inferencial la contrastación de hipótesis. 
http://doi.org/10.15359/ree.24-3.18

http://www.una.ac.cr/educare

educare@una.ac.cr

\section{Resultados y análisis}

Las variables de la investigación fueron la competencia comunicativa y la competencia intercultural, las cuales son variables cualitativas ordinales. Al ser un diseño de investigación experimental de tipo cuasiexperimental se compararan datos de dos muestras independientes (grupos de control y experimental). Para los cálculos estadísticos descriptivos e inferenciales se utilizó la herramienta informática IBM SPSS Statistics 24.

\section{Resultados descriptivos de la sensibilidad intercultural de la competencia intercultural}

Para esta investigación, se calculó la distribución de frecuencias, para luego determinar y comparar los porcentajes de los resultados pretest y postest, en el grupo de control y grupo experimental respectivamente.

En la Tabla 1 se observan los resultados descriptivos para el nivel de sensibilidad intercultural en el personal docente universitario.

Tabla 1: Nivel de competencias interculturales, niveles de sensibilidad intercultural de la competencia intercultural en el personal docente universitario del grupo de control (GC) y del grupo experimental (GE) para el pretest y postest

\begin{tabular}{lcccc}
\hline Nivel de sensibilidad intercultural & \multicolumn{2}{c}{$\mathrm{GC}(\mathrm{n}=20)$} & \multicolumn{2}{c}{$\mathrm{GE}(\mathrm{n}=20)$} \\
\hline & Pretest & & & \\
\hline & $\boldsymbol{f}_{\boldsymbol{i}}$ & $\%$ & $\boldsymbol{f}_{\boldsymbol{i}}$ & $\%$ \\
Poco adecuado & 2 & $10 \%$ & 3 & $15 \%$ \\
Adecuado & 8 & $40 \%$ & 8 & $40 \%$ \\
Muy adecuado & 10 & $50 \%$ & 9 & $45 \%$ \\
\hline & Postest & & & \\
\hline & $\boldsymbol{f}_{i}$ & $\%$ & $\boldsymbol{f}_{i}$ & $\%$ \\
Poco adecuado & 2 & $10 \%$ & 0 & $0 \%$ \\
Adecuado & 9 & $45 \%$ & 1 & $5 \%$ \\
Muy adecuado & 9 & $45 \%$ & 19 & $95 \%$ \\
\hline
\end{tabular}

Nota: Elaboración propia. 
http://doi.org/10.15359/ree.24-3.18

http://www.una.ac.cr/educare educare@una.ac.cr

En la Figura 1 se aprecian, gráficamente, los resultados descriptivos para el nivel de sensibilidad intercultural en el personal docente universitario.

Figura 1: Nivel de competencias interculturales, sensibilidad intercultural, GC y GE

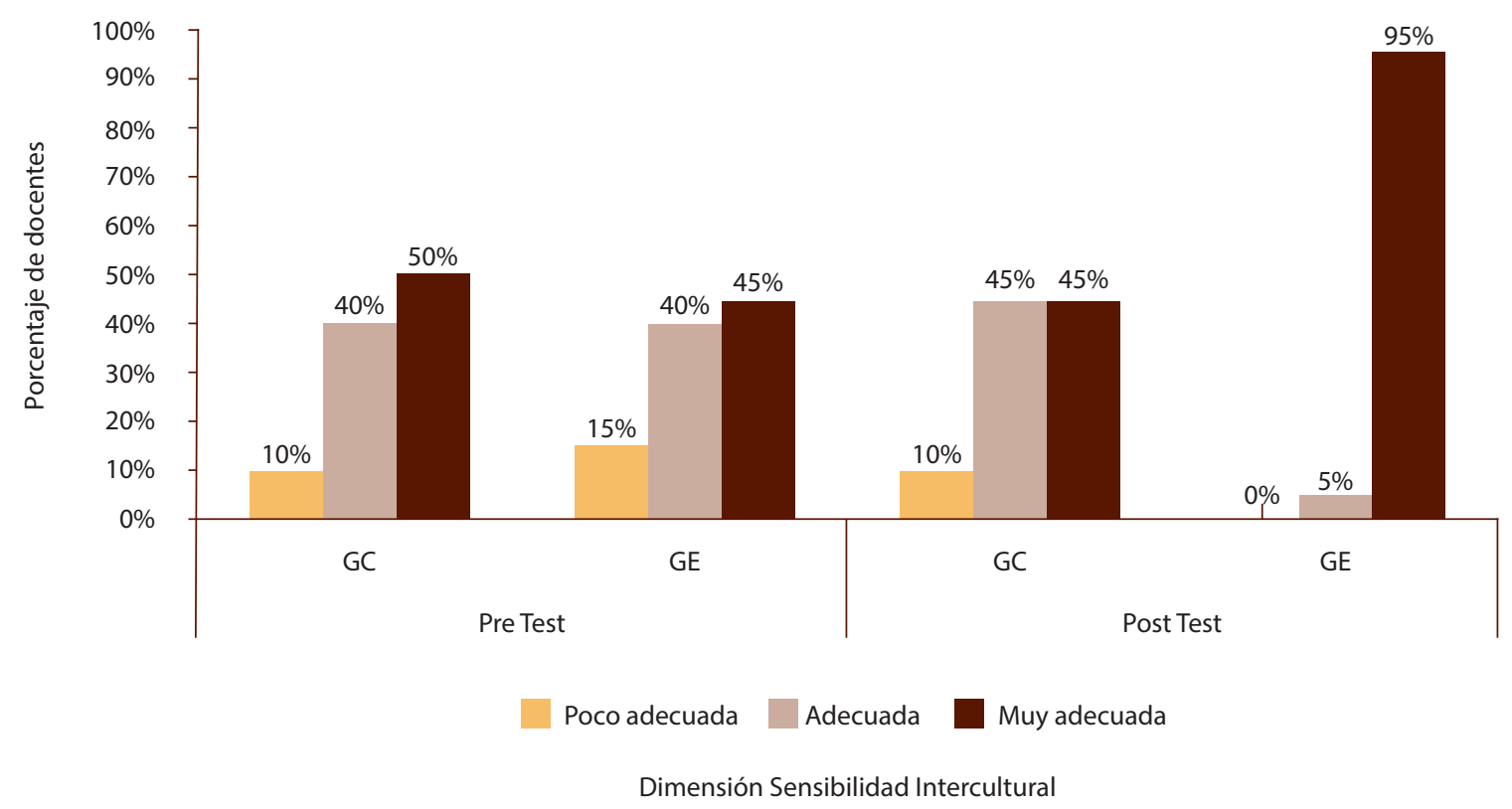

Nota: Elaboración propia.

\section{Análisis descriptivo de la sensibilidad intercultural de la competencia intercultural}

\section{Objetivo específico}

Determinar el efecto del taller "Comunicándonos" en la sensibilidad intercultural de las competencias interculturales en el personal docente de un área académico profesional de una universidad privada de Lima, 2018.

\section{Durante el pretest:}

De los valores incluidos en la Tabla 1 para el nivel de sensibilidad intercultural de las competencias interculturales en personal docente universitario de un área académica profesional de una Universidad privada de Lima, 2018, se apreciaron, tanto para el GC como 
http://doi.org/10.15359/ree.24-3.18

http://www.una.ac.cr/educare

educare@una.ac.cr

para el GE, resultados semejantes, se observó que entre el $10 \%$ y $15 \%$ del personal docente presenta un nivel de sensibilidad intercultural de poco adecuado, $40 \%$ adecuado, y entre $45 \%$ y 50 \% muy adecuado. Se concluye: el GC y GE presentan condiciones iniciales similares en los resultados del desarrollo sobre la sensibilidad intercultural de la competencia intercultural.

\section{Durante el postest:}

Posterior a la aplicación del taller "Comunicándonos", se observaron los valores incluidos en la Tabla 1 para el nivel de sensibilidad intercultural de las competencias interculturales en el personal docente universitario de un área académica profesional de una universidad privada de Lima, 2018, para el GC y para el GE, los cuales fueron distintos, pues en el GC el $45 \%$ del personal docente se encuentra en el nivel de muy adecuado, mientras que en el GE el $95 \%$ de docentes se encuentra en los niveles de muy adecuado. Se puede concluir que: el GC y el GE tienen diferencias en los resultados del desarrollo sobre sensibilidad intercultural de la competencia intercultural luego de aplicado el taller "Comunicándonos".

\section{Resultados inferenciales de la sensibilidad intercultural de la competencia intercultural}

Los datos fueron obtenidos mediante valores según escala de Likert, por lo que correspondió aplicar prueba estadística no paramétrica; asimismo, al ser un diseño de investigación experimental de tipo cuasi-experimental se compararon datos de dos muestras independientes (grupos de control y experimental), lo cual implica utilizar la Prueba de $U$ de Mann-Whitney.

\section{Prueba de hipótesis}

Hipótesis nula (Ho): $\mu 1=\mu 2$

La aplicación del taller "Comunicándonos" no tiene efecto en la sensibilidad intercultural de competencias interculturales en el personal docente universitario de un área académica profesional de una universidad privada de Lima, 2018.

Hipótesis alternativa (Ha): $\mu 1 \neq \mu 2$

La aplicación del taller "Comunicándonos" tiene efecto en la sensibilidad intercultural de competencias interculturales en el personal docente universitario de un área académica profesional de una universidad privada de Lima, 2018. 
http://doi.org/10.15359/ree.24-3.18

http://www.una.ac.cr/educare educare@una.ac.cr

Nivel de confianza: $95 \%$ (nivel de significancia $a=0,05$ )

Regla de decisión:

Si $p<a$, entonces se rechaza la Ho.

Si $\mathrm{p}>\mathrm{a}$, entonces se acepta la Ho.

Prueba estadística: Prueba de U de Mann-Whitney.

Tabla 2: Prueba U de Mann-Whitney con el fin de probar la hipótesis

\begin{tabular}{|c|c|c|c|c|c|c|}
\hline \multicolumn{5}{|c|}{ Rangos } & \multicolumn{2}{|c|}{ Estadísticos de contraste ${ }^{a}$} \\
\hline & Test y Grupo & $\mathrm{N}$ & Intervalo promedio & Adición de rangos & & \\
\hline \multirow{8}{*}{$\begin{array}{l}\text { Sensibilidad } \\
\text { intercultural }\end{array}$} & Pre test- GC & 20 & 21,55 & \multirow{2}{*}{431,00} & U de Mann-Whitney & 179,000 \\
\hline & & & & & W de Wilcoxon & 389,000 \\
\hline & Pre test- GE & 20 & 19,45 & \multirow{2}{*}{389,00} & Z &,- 573 \\
\hline & & & & & Sig. Asintót. (bilateral) &, 566 \\
\hline & \multirow[t]{2}{*}{ Pos test-GC } & \multirow[t]{2}{*}{20} & \multirow[t]{2}{*}{12,40} & \multirow{2}{*}{248,00} & U de Mann-Whitney & 38,000 \\
\hline & & & & & W de Wilcoxon & 248,000 \\
\hline & \multirow{2}{*}{ Pos test-GE } & \multirow{2}{*}{20} & \multirow{2}{*}{28,60} & \multirow{2}{*}{572,00} & Z & $-4,436$ \\
\hline & & & & & Sig. Asintót. (bilateral) &, 000 \\
\hline
\end{tabular}

a. Variable de agrupación: Test y grupo.

Nota: Elaboración propia

\section{Análisis inferencial de la sensibilidad intercultural de la competencia intercultural}

\section{Objetivo específico}

Determinar el efecto del taller "Comunicándonos" en la sensibilidad intercultural de las competencias interculturales en el personal docente universitario de un área académico profesional de una universidad privada de Lima, 2018. 
http://doi.org/10.15359/ree.24-3.18

http://www.una.ac.cr/educare

educare@una.ac.cr

\section{En el pretest:}

De los valores incluidos en la Tabla 2 para el nivel de la sensibilidad intercultural de las competencias interculturales no se observaron diferencias numéricas significativas en el intervalo promedio y en la adición de rangos entre el GC y GE; asimismo, en los estadísticos de los grupos de estudio, se apreció que el nivel de significancia $\mathbf{S i g}=\mathbf{0 , 0 0 0}$ es mayor que $a=0,05(p>a)$ y $\mathbf{Z}=-\mathbf{0 , 5 7 3}$ es mayor que -1.96 (punto crítico), por lo cual se concluye que el personal docente universitario al inicio presenta resultados semejantes en referencia al nivel de la sensibilidad intercultural de las competencias interculturales, por tanto no hay diferencias significativas entre el GC y GE.

\section{En el postest:}

De los valores incluidos en la Tabla 2 para el nivel la dimensión sensibilidad de competencias interculturales, si se mostraron diferencias numéricas significativas en el intervalo promedio $(12,40$ y 28,60$)$ y en la adición de rangos $(248,00$ y 572,00$)$ entre el GC y GE, en tal sentido, en los estadísticos de los referidos grupos, se encuentra que el nivel de significancia $\mathbf{S i g}=\mathbf{0 , 0 0 0}$ es menor que $a=0,05(p<a)$ y $\mathbf{Z}=\mathbf{- 4 , 4 3 6}$ es menor que -1.96 (punto crítico); es decir, sí hay diferencias significativas entre el GC y GE: La aplicación del taller "Comunicándonos" tiene un efecto positivo en el nivel de sensibilidad de las competencias interculturales del personal docente en un área académica profesional de una universidad privada de Lima, 2018.

\section{Discusión}

Se observa, de los resultados de la investigación de la Tabla 1, que aplicando el taller "Comunicándonos" existen diferencias en los resultados del desarrollo sobre sensibilidad intercultural de la competencia intercultural en personal docente universitario de un área académica profesional de una universidad privada de Lima, 2018. Al respecto, Byram (1997) sostiene que la competencia comunicativa intercultural implica la habilidad de comprender y relacionarse con personas de otros países pretendiendo brindar condiciones favorables de la comunicación intercultural. En ese sentido, en Competencias interculturales. Diversidad cultural (2018) indicó que las competencias interculturales están compuestas por las habilidades cognitivas, afectivas y prácticas, las cuales son importantes para interrelacionarse en un contexto intercultural. Estas capacidades son necesarias para propiciar un ámbito pedagógico en el cual el estudiantado sea aceptado y atendido por sus propias habilidades; y para permitir la interacción eficiente y equitativa de la totalidad de sus miembros. Asimismo, se concuerda con Meyer (1991), en que la competencia intercultural es parte de una amplia competencia del sujeto hablante de una lengua extranjera, identificada con la habilidad de una persona de actuar de forma adecuada y flexible al enfrentarse con acciones, actitudes y expectativas de personas de otras culturas. 
http://doi.org/10.15359/ree.24-3.18 http://www.una.ac.cr/educare educare@una.ac.cr

De otro lado, los resultados de la investigación de la Tabla 2, dan cuenta de que la aplicación del taller "Comunicándonos" sí tuvo efecto positivo en la sensibilidad intercultural de las competencias interculturales en personal docente universitario de un área académica profesional de una universidad privada de Lima, 2018. Concordante con estos resultados, Ricardo Barreto (2013) demuestra que el desarrollo de actividades basadas en el reconocimiento a la realidad social y contextual es una oportunidad para que el alumnado reflexione sobre sus actividades desde diferentes perspectivas, desde diferentes culturas y desde diversos tópicos disciplinares; fomenta y difunde la integración con la evaluación del aprendizaje y un ambiente adecuado para el quehacer colaborativo. Del mismo modo, Sanhueza Henríquez (2010) señala las sensibilidades interculturales comolas capacidadesque tienen las personas para emitir respuestas emocionales positivas y controlar aquellas emociones que pueden perjudicar el proceso comunicativo intercultural, lo cual verifica los resultados de Illescas García (2015) que afirmó, respecto a la relevancia del tema intercultural en la enseñanza de un segundo idioma con la finalidad que el alumnado pueda interactuar con las personas hablantes nativas en igualdad de condiciones, la premisa de que lengua y cultura no se pueden disociar. Esta sigue siendo válida y lo cual quedó manifestado a lo largo del trabajo.

De otro parte, se ha observado que la aplicación del taller "Comunicándonos" en el personal docente universitario de un área académica profesional de una universidad privada de Lima, 2018, se ha realizado de manera participativa, en un ambiente que facilitó la enseñanza y aprendizaje del personal docente. Al respecto, Arias-Gómez et al. (2016) sostienen que los talleres son espacios en los cuales se aprende haciendo de una manera práctica.

\section{Conclusiones}

La aplicación del taller "Comunicándonos" provocó la existencia de diferencias en los resultados del desarrollo sobre sensibilidad intercultural de las competencias interculturales en el personal docente universitario de un área académica profesional de una universidad privada de Lima, 2018.

La aplicación del taller "Comunicándonos" impactó positivamente en la sensibilidad intercultural de las competencias interculturales en el personal docente universitario de un área educativa profesional de una universidad privada de Lima, 2018.

A modo de reflexión, considero que el taller "Comunicándonos" efectuado debería ser aplicado no solo en otras áreas académico profesional de la universidad del presente estudio, sino en otras universidades, con el fin de fortalecer las competencias interculturales del personal docente universitario, en las dimensiones de conciencia intercultural, habilidad intercultural y sensibilidad intercultural, sin perjuicio de una reconfiguración adecuada a cada contexto, en particular en los modelos de comunicación intercultural, y materializándolos con formas de representación tales como actividades, gráficos, tablas y símbolos para la demostración de lo aprendido. 
http://doi.org/10.15359/ree.24-3.18

http://www.una.ac.cr/educare

educare@una.ac.cr

Finalmente, recomiendo que esta investigación sea difundida con el fin de servir de referente para futuras propuestas para la mejora de los currículos formativos del personal docente universitario, para así fortalecer la calidad educativa.

\section{Declaración de Material complementario}

Este artículo tiene disponible como material complementario:

- La versión preprint del artículo en https://doi.org/10.5281/zenodo.3359338

\section{Referencias}

Aneas Álvarez, M. A. (2005). Competencia intercultural, concepto, efectos e implicaciones en el ejercicio de la ciudadanía. Revista Iberoamericana de Educación, 36(13), 1-10. https://doi. org/10.35362/rie36133004

Arias-Gómez, J., Villasís-Keever M. Á., Miranda-Novales M. G. (2016). El protocolo de investigación III:La población de estudio. RevistaAlergia México, 63(2),201-206. http://www.revistaalergia. mx/ojs/index.php/ram/article/view/181/273

Byram, M. (1997). Teaching and assessing intercultural communicative competence. Multilingual Matters.

Careaga, A., Sica, R., Cirillo, A. y Da Luz, S. (2006). Aportes para diseñar e implementar un taller. $8^{\text {vo }}$ Seminario-Taller en Desarrollo Profesional Médico Continuo, 2 das Jornadas de Experiencias educativas en DPMC.

Consorcio Intercultural. (2007). Reflexiones de Raúl Fornet-Betancourt. Sobre el concepto de interculturalidad (2. ${ }^{a}$ reimp.). Coordinación General de Educación Intercultural y Bilingüe.

Competencias interculturales. Diversidad cultural (18 de abril, 2013). [Archivo en un blog]. Blog El espacio de la diversidad. https://elespaciodeladiversidad.blogspot.com/2013/04/ competencias-interculturales-diversidad.html

de Vallescar Palanca, D. (2000). Cultura, multiculturalismo e interculturalidad: Hacia una racionalidad intercultural [Tesis doctoral]. Universidad Complutense de Madrid.

Estermann, J. (2010). Interculturalidad: Vivir la diversidad. ISEAT.

Gastulo Jiménez, Y. N., Maquen Niño, E. J. C. y Severino Ordoñez, M. (2018). Modelo holístico configuracional para el desarrollo de la competencia intercultural en los docentes de educación secundaria en Motupe - Perú. Revista Científica Universidad Señor de Sipán, 10(2), 199-212. https://doi.org/10.26495/rtzh1810.225913

Guadamillas Gómez, M. V. (2014). El desarrollo de la competencia comunicativa e intercultural a través de recursos creativos: Análisis de la poesía, el teatro y el cómic como materiales 
http://doi.org/10.15359/ree.24-3.18

http://www.una.ac.cr/educare educare@una.ac.cr

didácticos en la enseñanza de inglés como lengua extranjera [Tesis doctoral]. Universidad de Castilla-La Mancha, España. https://ruidera.uclm.es/xmlui/handle/10578/6551

Illescas García, A. (2015). La competencia intercultural en la enseñanza de ELE: Trabajo de campo sobre manuales y estudiantes de español [Tesis doctoral]. Universidad de Granada, España. https://digibug.ugr.es/handle/10481/40377

Jandt, F. E. (1995). Intercultural communication: An introduction. Sage.

Martínez-Lirola, M. (2018). La importancia de introducir la competencia intercultural en la educación superior: Propuesta de actividades prácticas. Revista Electrónica Educare, 22(1), 1-19. https://doi.org/10.15359/ree.22-1.3

Meyer, M. (1991). Developing transcultural competence: Case studies of advanced foreign language learners. En D. Buttjes y M. Byram (Eds.), Mediating languages and csultures (pp. 136-158). Multilingual Matters.

Morales-Saavedra, S., Quintriqueo-Millán, S., Uribe-Sepúlveda, P. A. y Arias-Ortega, K. (2018). Interculturalidad en educación superior: Experiencia en educación inicial en La Araucanía, Chile. Convergencia Revista de Ciencias Sociales, 77, 55-76. https://doi.org/10.29101/crcs. v25i77.4706

Preiswerk M. (2011). Contrato intercultural: Crisis y refundación de la educación teológica. Plural Editores.

Ricardo Barreto, C. T. (2013). Formación y desarrollo de la competencia intercultural en ambientes virtuales de aprendizaje [Tesis Doctoral]. Universidad Nacional de Educación a Distancia, España. http://e-spacio.uned.es/fez/view.php?pid=tesisuned:Educacion-Ctricardo

Salmona, M., Partlo, M., Kaczynski, D. y Leonard, S. (2015). Developing culturally competent teachers: An international student teaching field experience. Australian Journal of Teacher Education, 40(4), 34-53. https://doi.org/10.14221/ajte.2015v40n4.3

Sanhueza Henríquez, S. V. (2010). Sensibilidad intercultural: Un estudio exploratorio con alumnado de educación primaria y secundaria en la provincia de Alicante [Tesis doctoral]. Universidad de Alicante, España. http://rua.ua.es/dspace/handle/10045/18332

Sanhueza Henríquez, S., Patrick Matzler, P., Hsu, C.-C., Dominguez Maldonado, J., Friz Carrillo, M. y Quintriqueo Millán, S. (2016). Competencias comunicativas interculturales en la formación inicial docente: El caso de tres universidades regionales de Chile. Estudios Pedagógicos, 42(4) 183-200. http://dx.doi.org/10.4067/S0718-07052016000500011

Sanhueza Henríquez, S., Paukner Nogués, F., San Martín, V. y Friz Carrillo, M. (2012). Dimensiones de la competencia comunicativa intercultural $(\mathrm{CCl})$ y sus implicaciones para la práctica educativa. Folios, 36, 131-151. https://doi.org/10.17227/01234870.36folios131.151 
http://doi.org/10.15359/ree.24-3.18

http://www.una.ac.cr/educare

educare@una.ac.cr

Apéndice A: Cuestionario sobre el nivel de la dimensión sensibilidad intercultural de la competencia intercultural en el personal docente universitario

Cada docente universitario respondió al ítem presentado marcando con una " $\mathrm{X}$ " en el recuadro respectivo.

Por ejemplo, se tiene la siguiente pregunta:

\begin{tabular}{|c|c|c|c|c|c|c|}
\hline \multirow{2}{*}{$\mathbf{N}^{\circ}$} & \multicolumn{5}{c|}{ Respuestas } \\
\cline { 3 - 7 } & ITEMS & $\mathbf{1}$ & $\mathbf{2}$ & $\mathbf{3}$ & $\mathbf{4}$ & $\mathbf{5}$ \\
\hline 01 & Presento información basada en bibliografía. & & & & $\mathrm{X}$ & \\
\hline
\end{tabular}
1. Nunca
2. Casi Nunca
3. Algunas veces
4. Casi siempre
5. Siempre

A continuación, se presenta el cuestionario:

\begin{tabular}{|c|c|c|c|c|c|c|}
\hline \multirow{2}{*}{$\mathbf{N}^{\circ}$} & \multirow{2}{*}{ DIMENSIÓN / ITEMS } & \multicolumn{5}{|c|}{ Respuestas } \\
\hline & & 1 & 2 & 3 & 4 & 5 \\
\hline \multicolumn{7}{|c|}{ DIMENSIÓN SENSIBILIDAD INTERCULTURAL } \\
\hline 01 & $\begin{array}{l}\text { Considero que el manejo afectivo es importante en una efectiva interrelación } \\
\text { intercultural. }\end{array}$ & & & & & \\
\hline 02 & $\begin{array}{l}\text { Promuevo el respeto de las ideas y opiniones de los otros en las interacciones } \\
\text { interculturales teniendo en cuenta que son parte del aprendizaje. }\end{array}$ & & & & & \\
\hline 03 & $\begin{array}{l}\text { Considero que la apertura de mente favorece la aceptación de las explicaciones de } \\
\text { los demás. }\end{array}$ & & & & & \\
\hline 04 & $\begin{array}{l}\text { Considero que la apertura de mente se fortalece con el reconocimiento de ideas de } \\
\text { otras culturas. }\end{array}$ & & & & & \\
\hline 05 & $\begin{array}{l}\text { Trato de percibir las expresiones de las relaciones interculturales como fuente de un } \\
\text { aprendizaje integrado. }\end{array}$ & & & & & \\
\hline 06 & $\begin{array}{l}\text { Me esfuerzo por ser empático con la persona culturalmente distinto para entenderlo } \\
\text { mejor. }\end{array}$ & & & & & \\
\hline 07 & Desarrollo pensamientos en la interacción intercultural es útil en la enseñanza. & & & & & \\
\hline 08 & Considero que la autorregulación promueve las interacciones interculturales. & & & & & \\
\hline 09 & $\begin{array}{l}\text { Cuido en cambiar mis propios comportamientos con el fin de mejorar las } \\
\text { interacciones interculturales, con el consiguiente aprendizaje. }\end{array}$ & & & & & \\
\hline 10 & $\begin{array}{l}\text { Considero que las interacciones culturales requieren sensibilidad, atención y } \\
\text { perspicacia del docente. }\end{array}$ & & & & & \\
\hline
\end{tabular}

Los ítems están basados en Sanhueza Henríquez et al. (2012). 
http://doi.org/10.15359/ree.24-3.18

Apéndice B: Temática del taller "Comunicándonos"

\begin{tabular}{|c|c|c|}
\hline Sesión 1 & Sesión 2 & Sesión 3 \\
\hline $\begin{array}{l}\text { Competencias comunicativas del } \\
\text { personal docente universitario I }\end{array}$ & $\begin{array}{l}\text { Competencias comunicativas del } \\
\text { personal docente universitario II }\end{array}$ & $\begin{array}{l}\text { Competencias comunicativas del } \\
\text { personal docente universitario III }\end{array}$ \\
\hline - Componente cognitivo & - Componente comunicativo & - Componente sociocultural \\
\hline $\begin{array}{l}\text { - Conocimientos sobre } \\
\text { comprensión y construcción de } \\
\text { significados. } \\
\text { - Habilidades para la comprensión } \\
\text { y construcción de significados. } \\
\text { - Actitudes relacionadas con la } \\
\text { comprensión y construcción de } \\
\text { significados. }\end{array}$ & $\begin{array}{l}\text { - Conocimientos sobre el proceso } \\
\text { de comunicación humana. } \\
\text { - Habilidades para la comunicación } \\
\text { humana, para expresar, transmitir } \\
\text { y recibir mensajes de naturaleza } \\
\text { verbal y no verbal. } \\
\text { - Actitudes positivas en la } \\
\text { relación con el estudiante. }\end{array}$ & $\begin{array}{l}\text { - Conocimientos sobre el } \\
\text { contexto. } \\
\text { - Habilidades para adecuarse al } \\
\text { contexto. } \\
\text { - Actitudes relacionadas } \\
\text { con el desenvolvimiento } \\
\text { en diferentes contextos } \\
\text { comunicativos. }\end{array}$ \\
\hline Dinámica 1: & Dinámica 2: & Dinámica 3: \\
\hline $\begin{array}{l}\text { El semáforo - Importancia del } \\
\text { componente cognitiv }\end{array}$ & $\begin{array}{l}\text { Actividades comunicativas del } \\
\text { docente }\end{array}$ & $\begin{array}{l}\text { Conociendo el contexto - El } \\
\text { equipo pequeño }\end{array}$ \\
\hline Estudio de caso 1: & Estudio de caso 2: & Estudio de caso 3: \\
\hline $\begin{array}{l}\text { ¿Cuánto valoro la información } \\
\text { recibida del estudiante? }\end{array}$ & $\begin{array}{l}\text { ¿Cuáles son las actitudes en tu } \\
\text { comunicación? }\end{array}$ & $\begin{array}{l}\text { ¿Cuáles son las actitudes con tus } \\
\text { alumnos? }\end{array}$ \\
\hline Sesión 4 & Sesión 5 & Sesión 6 \\
\hline $\begin{array}{l}\text { Competencias interculturales del } \\
\text { docente universitario I }\end{array}$ & $\begin{array}{l}\text { Competencias interculturales del } \\
\text { docente universitario II }\end{array}$ & $\begin{array}{l}\text { Competencias interculturales del } \\
\text { docente universitario III }\end{array}$ \\
\hline - Conciencia intercultural & - Habilidad intercultural & - Sensibilidad intercultural \\
\hline $\begin{array}{l}\text { - Comprensión de las } \\
\text { características distintivas de su } \\
\text { cultura. } \\
\text { - Comprensión de las } \\
\text { características de } \\
\text { - otras culturas. }\end{array}$ & $\begin{array}{l}\text { - Flexibilidad del } \\
\text { comportamiento. } \\
\text { - Manejo de la interacción. }\end{array}$ & $\begin{array}{l}\text { - Autoconcepto. } \\
\text { - Apertura de mente. } \\
\text { - Actitudes de no juzgar. } \\
\text { - Empatía. } \\
\text { - Autorregulación. } \\
\text { - Implicación en la interacción. }\end{array}$ \\
\hline Dinámica 4: & Dinámica 5: & Dinámica 6: \\
\hline $\begin{array}{l}\text { Conociendo las características } \\
\text { culturales de mis estudiantes. }\end{array}$ & Bafa: Juego de roles culturales. & $\begin{array}{l}\text { Socializando las conclusiones } \\
\text { relevantes de la sensibilidad } \\
\text { intercultural en las competencias } \\
\text { interculturales de docentes. }\end{array}$ \\
\hline Estudio de caso 4: & Estudio de caso 5: & Estudio de caso 6: \\
\hline $\begin{array}{l}\text { ¿Cuánto valoro las características } \\
\text { distintivas de mi cultura respecto } \\
\text { a otras culturas? }\end{array}$ & $\begin{array}{l}\text { ¿Cómo podría promover reuniones } \\
\text { eficaces interculturales? }\end{array}$ & $\begin{array}{l}\text { ¿Considero que la apertura de } \\
\text { mente favorece el aprendizaje } \\
\text { intercultural? }\end{array}$ \\
\hline
\end{tabular}

